Rakenteiden Mekaniikka (Journal of Structural Mechanics)

Vol. 52, No 2, 2019, pp. 53-60

http://rakenteidenmekaniikka.journal.fi/index

https://doi.org/10.23998/rm.75287

(C) 2019 The Authors

Open access under license CC BY 4.0

\title{
Numerical modelling of heat generation during shear band formation in rock
}

\author{
Timo Saksala ${ }^{1}$, Martina Pressacco, Sami Holopainen and Reijo Kouhia
}

\begin{abstract}
Summary. This article gives a computational continuum mechanics answer to a question of how much heat is generated, in terms of temperature rise, during controlled shear band formation in a rock like material. This problem is treated as adiabatic heating due to mechanical dissipation at the material point level. Assuming that only the compressive strength of the rock is temperature dependent, the coupled system of the constitutive equations and the adiabatic heat equation can be solved as a second order polynomial equation for the viscoplastic multiplier at an integration point. A Mohr-Coulomb viscoplastic model with linear softening is employed for rock material description. Numerical simulations of a 2D strip under uniaxial compression at strain rates up to $10 \mathrm{1} / \mathrm{s}$ show that the temperature rise in a rock like material with a compressive strength of $100 \mathrm{MPa}$ is less than two degrees.
\end{abstract}

Keywords: adiabatic heating, mechanical dissipation, shear band, rock fracture, finite element method

Received 1 October 2018. Accepted 12 December 2018. Published online 16 August 2019.

\section{Introduction}

Inelastic deformation and damage processes in quasi-brittle materials are usually assumed to be isothermal. While this assumption is justifiable, especially under quasistatic loading, it is still interesting to know how much heat is generated during fracture/inelastic failure processes of quasi-brittle materials such as rock and concrete, especially under dynamic loading. For example, if we are to solve a coupled thermoplasticity problem of rock fracture due to a thermal shock by an external heat flux, we want to know a-priori whether the mechanical heating effects can be neglected as insignificant in comparison to the effects of the external heat flux.

${ }^{1}$ Corresponding author: timo.saksala@tut.fi 
This problem is dealt with in this paper. Namely, we give a computational mechanics answer to the question of how much heat, in the sense of temperature rise, is generated in a controlled shear band formation in rock material under compression. This kind of problem is quite of often studied related to metals but seldom addressed in the field of rock mechanics. We mention here the study by Ben-Zion and Sammis [1], which investigates the shear heating in geological faults involving distributed fracturing and pulverization of rocks. Their simple calculations predict that the temperature rise could be even $100^{\circ} \mathrm{C}$ in depths from 1 to $5 \mathrm{~km}$ due to intensive shearing involving inelastic strains ranging from 0.4 to 0.8 . However, much milder conditions are considered in the present study, i.e. unconfined compression at normal conditions (at room temperature and atmospheric pressure).

In this study, a Mohr-Coulomb viscoplasticity model for rock, augmented with the adiabatic heat equation, is employed and implemented in the finite element method for solving the problem of adiabatic heating during shear band formation in rock. In the numerical examples, a 2D strip made of rock under uniaxial compression at different strain rates is simulated in order to answer the research question posed above.

\section{Numerical model}

All the theoretical and numerical considerations are carried out here under the small deformation assumption enabling the additive split of the total strain into elastic, viscoplastic and thermal parts by $\boldsymbol{\varepsilon}=\boldsymbol{\varepsilon}_{\mathrm{e}}+\boldsymbol{\varepsilon}_{\mathrm{vp}}+\boldsymbol{\varepsilon}_{\theta}$.

According to Ottosen and Ristinmaa [2], adiabatic heating conditions are such that neither heat exchange with the surroundings of the body nor heat flow in the body can occur due to rapid loading. In these circumstances, there is no external heat sources and, thus, the related thermo-mechanical problem can be solved at the material point level, along with the constitutive equations, since the only heat source is the mechanical coupling.

The problem of heat generation during shear banding in rock is such a problem. Thereby, it is solved with a simple thermo-viscoplasticity model for rock. We choose the Mohr-Coulomb yield criterion with a linear softening law for the compressive strength as the basis for a viscoplastic consistency model for rock. The Young's modulus and yield strength are assumed to depend linearly on temperature. The model thus specified can be written as

$$
\begin{aligned}
& f_{\mathrm{MC}}(\boldsymbol{\sigma}, \kappa, \dot{\kappa}, \theta)=\underbrace{k_{\varphi} \sigma_{1}-\sigma_{3}}_{\hat{f}_{\mathrm{MC}}(\boldsymbol{\sigma})}-\left(\sigma_{\mathrm{c}}(\kappa, \dot{\kappa})+K_{\theta}\left(\theta-\theta_{0}\right)\right) \\
& \sigma_{\mathrm{c}}(\kappa, \dot{\kappa})=\sigma_{\mathrm{c} 0}+K_{\mathrm{m}} \kappa+s \dot{\kappa}, \quad k_{\varphi}=(1+\sin \varphi) /(1-\sin \varphi) \\
& \dot{\boldsymbol{\varepsilon}}_{\mathrm{vp}}=\dot{\lambda} \frac{\partial g_{\mathrm{MC}}}{\partial \boldsymbol{\sigma}}, \quad \dot{\kappa}=-\dot{\lambda} \frac{\partial g_{\mathrm{MC}}}{\partial q} \equiv \dot{\lambda}, \quad E(\theta)=E_{0}+K_{\theta}^{E}\left(\theta-\theta_{0}\right) \\
& g_{\mathrm{MC}}(\boldsymbol{\sigma})=k_{\psi} \sigma_{1}-\sigma_{3}-\left(\sigma_{\mathrm{c} 0}+q\right), \quad k_{\psi}=(1+\sin \psi) /(1-\sin \psi) \\
& f_{\mathrm{MC}} \leq 0, \quad \dot{\lambda} \geq 0, \quad \dot{\lambda} f_{\mathrm{MC}}=0
\end{aligned}
$$


where $\boldsymbol{\sigma}$ is the stress tensor, $\kappa, \dot{\kappa}$ are the internal variable and its rate, $\theta$ and $\theta_{0}$ are the present and reference temperature, $\sigma_{1}, \sigma_{3}$ are the minor and major principal stresses, respectively, $q$ is the stress like softening parameter, $\sigma_{\mathrm{c} 0}$ is the initial compressive strength, $K_{\theta}, K_{\mathrm{m}}$ are the thermal and mechanical softening modulus for compressive strength, respectively, and $K_{\theta}^{E}$ is the thermal softening modulus for Young's modulus $E$. Moreover, $s$ is the constant viscosity modulus, $\varphi$ and $\psi$ are the internal friction and dilation angle, respectively, $\dot{\boldsymbol{\varepsilon}}_{\mathrm{vp}}$ is the viscoplastic strain rate (tensor) depending on the plastic potential $g_{\mathrm{MC}}$, accounting for nonassociative flow, and viscoplastic increment $\dot{\lambda}$. Finally, the last equations in (1) define the consistency conditions. The present model is thus written in the viscoplastic consistency format by Wang et al. [3].

We still need the heat equation for temperature evolution description. In adiabatic conditions (the heat sources as well as the gradient of the heat flux are ignored) can be written as [2]

$$
\begin{aligned}
& \rho c \dot{\theta}=\boldsymbol{\sigma}: \dot{\boldsymbol{\varepsilon}}_{\mathrm{vp}}-q \dot{\kappa}-\underbrace{\theta \mathbf{P}:\left(\dot{\boldsymbol{\varepsilon}}-\dot{\boldsymbol{\varepsilon}}_{\mathrm{vp}}\right)}_{\approx 0}+\theta \frac{\partial q}{\partial \theta} \dot{\kappa}=\dot{\lambda} \boldsymbol{\sigma}: \frac{\partial g_{\mathrm{MC}}}{\partial \boldsymbol{\sigma}}-K_{\mathrm{m}} \lambda \dot{\lambda} \\
& \mathbf{P}=\boldsymbol{\beta}+\frac{d \boldsymbol{\beta}}{d \theta}\left(\theta-\theta_{0}\right)-\frac{d \mathbf{E}}{d \theta}:\left(\boldsymbol{\varepsilon}-\boldsymbol{\varepsilon}_{\mathrm{vp}}\right), \quad \boldsymbol{\beta}=\alpha \mathbf{E}: \mathbf{1}
\end{aligned}
$$

where $\rho, c$ are the density and the specific heat capacity of the material, $\mathbf{E}$ is the elasticity tensor, $\boldsymbol{\varepsilon}$ is the total strain tensor, $\alpha$ is the thermal expansion coefficient, and $\mathbf{1}$ is the second order identity tensor. The fourth term in the right hand side is zero because the stress-like softening parameter does not, in this study, depend on the temperature. Moreover, the thermo-elasticity effects, i.e. the third term in (2), can be neglected as insignificant in comparison to thermo-plasticity effects. The effect of the third term, being directly proportional to the elastic strain rate, is at its greatest just below the elastic limit, i.e. the compressive strength, beyond which its effect diminishes as the viscoplastic strain increases. Simplifying Equation (2) in the elastic regime leads to the Kelvin's expression of thermo-elasticity as $\theta-\theta_{0}=-\alpha \theta_{0} \sigma_{k k} / \rho c$ [2]. Using this to estimate the temperature rise in a rock due to a sudden increase of compressive stress to $-100 \mathrm{MPa}$ gives $\Delta \theta=0.16{ }^{\circ} \mathrm{C}$ for the material properties given later. Therefore, the thermo-elastic effects can be ignored.

A glance at equations (1) and (2) reveals that the only independent variable to be solved is $\dot{\lambda}$. Therefore, as the consistency must be fulfilled at the end of a time step, we can proceed as follows: 


$$
\begin{aligned}
0 & =f_{\mathrm{MC}}\left(\boldsymbol{\sigma}_{n+1}, \lambda_{n+1}, \dot{\lambda}_{n+1}, \theta_{n+1}\right)=\underbrace{\frac{\partial \hat{f}_{\mathrm{MC}}(\boldsymbol{\sigma})}{\partial \boldsymbol{\sigma}}: \boldsymbol{\sigma}_{n+1}}_{\hat{f}_{\mathrm{MC}}(\boldsymbol{\sigma})}-\left(\sigma_{\mathrm{c} 0}+K_{\mathrm{m}} \lambda_{n+1}+s \dot{\lambda}_{n+1}+K_{\theta}\left(\theta_{n+1}-\theta_{0}\right)\right) \\
& =\frac{\partial \hat{f}_{\mathrm{MC}}(\boldsymbol{\sigma})}{\partial \boldsymbol{\sigma}}:\left(\boldsymbol{\sigma}_{n+1}^{\text {trial }}+\Delta \boldsymbol{\sigma}\right)-\left(\sigma_{\mathrm{c} 0}+K_{\mathrm{m}}\left(\lambda_{n}+\Delta \lambda\right)+s \frac{\lambda_{n}+\Delta \lambda}{\Delta t}+K_{\theta}\left(\theta_{n}+\Delta \theta-\theta_{0}\right)\right) \\
& =\underbrace{\frac{\partial \hat{f}_{\mathrm{MC}}(\boldsymbol{\sigma})}{\partial \boldsymbol{\sigma}}: \boldsymbol{\sigma}_{n+1}^{\text {trial }}}_{f_{\mathrm{MC}}^{\text {tial }}}-\left(\sigma_{\mathrm{c} 0}+K_{\mathrm{m}} \lambda_{n}+s \frac{\lambda_{n}}{\Delta t}+K_{\theta}\left(\theta_{n}-\theta_{0}\right)\right) \\
& \left.+\frac{\left.\partial \hat{f}_{\mathrm{MC}}^{\text {trial }}\right)}{\partial \boldsymbol{\sigma}}: \Delta \boldsymbol{\sigma}\right)
\end{aligned}
$$

where the fact that $\hat{f}_{\mathrm{MC}}$ is a homogeneous function of degree one and the definition of the trial stress according to the standard elastic predictor-plastic corrector split (see [4]) concept has been used. Moreover, the rate of viscoplastic increment has been replaced by its algorithmic counterpart: $\dot{\lambda}_{n+1}=\left(\lambda_{n}+\Delta \lambda\right) / \Delta t$. Now, the stress increment and the temperature increment can be expressed as

$$
\begin{aligned}
\Delta \boldsymbol{\sigma} & =-\mathbf{E}(\theta): \Delta \boldsymbol{\varepsilon}_{\mathrm{vp}}=-\Delta \lambda \mathbf{E}(\theta): \frac{\partial g_{\mathrm{MC}}}{\partial \boldsymbol{\sigma}} \text { and } \\
\Delta \theta & =\frac{1}{\rho c}\left(\boldsymbol{\sigma}_{n+1}: \Delta \boldsymbol{\varepsilon}_{\mathrm{vp}}-K_{\mathrm{m}} \lambda_{n+1} \Delta \lambda\right) \\
& =\frac{1}{\rho c}\left(\Delta \lambda \boldsymbol{\sigma}_{n+1}^{\text {trial }}: \frac{\partial g_{\mathrm{MC}}}{\partial \boldsymbol{\sigma}}-\Delta \lambda^{2} \frac{\partial g_{\mathrm{MC}}}{\partial \boldsymbol{\sigma}}: \mathbf{E}(\theta): \frac{\partial g_{\mathrm{MC}}}{\partial \boldsymbol{\sigma}}-K_{\mathrm{m}} \lambda_{n} \Delta \lambda-K_{\mathrm{m}} \Delta \lambda^{2}\right)
\end{aligned}
$$

Substituting these into (3), we obtain the second order equation for solving the viscoplastic increment:

$$
\begin{aligned}
& 0=A \Delta \lambda^{2}+B \Delta \lambda+C \text { with } \\
& A=\frac{K_{\theta}}{\rho c}\left(\frac{\partial g_{\mathrm{MC}}}{\partial \boldsymbol{\sigma}}: \mathbf{E}(\theta): \frac{\partial g_{\mathrm{MC}}}{\partial \boldsymbol{\sigma}}+K_{\mathrm{m}}\right) \\
& B=-\left(\frac{\partial \hat{f}_{\mathrm{MC}}(\boldsymbol{\sigma})}{\partial \boldsymbol{\sigma}}: \mathbf{E}(\theta): \frac{\partial g_{\mathrm{MC}}}{\partial \boldsymbol{\sigma}}+K_{\mathrm{m}}+\frac{s}{\Delta t}+\frac{K_{\theta}}{\rho c}\left(\boldsymbol{\sigma}_{n+1}^{\text {trial }}: \frac{\partial g_{\mathrm{MC}}}{\partial \boldsymbol{\sigma}}-K_{\mathrm{m}} \lambda_{n}\right)\right) \\
& C=f_{\mathrm{MC}}^{\text {trial }}
\end{aligned}
$$

Thus, at the integration point, the problem of adiabatic heat generation due to shear banding is reduced to solving a second degree equation for the viscoplastic multiplier. However, this result is valid only for this specific case where the temperature dependence of the Young's modulus is kept constant during the stress integration. The stress return mapping can now be sketched as 


$$
\begin{aligned}
& f_{\mathrm{MC}}^{\text {trial }}\left(\boldsymbol{\sigma}_{n+1}^{\text {trial }}, \lambda_{n}, \dot{\lambda}_{n}, \theta_{n}\right), \quad \boldsymbol{\sigma}_{n+1}^{\text {trial }}=\mathbf{E}\left(\theta_{n}\right):\left(\boldsymbol{\varepsilon}_{n+1}-\boldsymbol{\varepsilon}_{n}^{\mathrm{vp}}-\boldsymbol{\varepsilon}_{n}^{\theta}\right), \quad \boldsymbol{\varepsilon}_{n}^{\theta}=\alpha\left(\theta_{n}-\theta_{0}\right) \mathbf{I} \\
& \text { While } f_{\mathrm{MC}}^{k+1}=T O L \quad \text { Do } \\
& \text { Solve } 0=A \delta \lambda^{2}+B \delta \lambda+C \text { for } \delta \lambda \\
& \Delta \lambda_{k+1}=\Delta \lambda_{k}+\delta \lambda, \quad \dot{\lambda}_{k+1}=\frac{\Delta \lambda_{k+1}}{\Delta t}, \quad \lambda_{k+1}=\lambda_{k}+\Delta \lambda_{k+1} \\
& \delta \boldsymbol{\varepsilon}_{\mathrm{vp}}=\delta \lambda \frac{\partial g_{\mathrm{MC}}}{\partial \boldsymbol{\sigma}}, \quad \boldsymbol{\varepsilon}_{\mathrm{vp}}^{k+1}=\boldsymbol{\varepsilon}_{\mathrm{vp}}^{k}+\delta \boldsymbol{\varepsilon}_{\mathrm{vp}} \\
& \boldsymbol{\sigma}_{k+1}=\boldsymbol{\sigma}_{k}-\mathbf{E}\left(\theta_{n}\right): \delta \boldsymbol{\varepsilon}_{\mathrm{vp}} \\
& \Delta \theta=\frac{\delta \lambda}{\rho c}\left(\boldsymbol{\sigma}_{k+1}: \frac{\partial g_{\mathrm{MC}}}{\partial \boldsymbol{\sigma}}-K_{\mathrm{m}} \lambda_{k+1}\right), \quad \theta_{k+1}=\theta_{k}+\Delta \theta \\
& f_{\mathrm{MC}}^{k+1}=f_{\mathrm{MC}}\left(\boldsymbol{\sigma}_{k+1}, \lambda_{k+1}, \dot{\lambda}_{k+1}, \theta_{k+1}\right)
\end{aligned}
$$

However, the Young's modulus is kept constant during the stress integration and updated only after solving the temperature by $E_{n+1}=E_{0}+K_{\theta}^{E}\left(\theta_{n+1}-\theta_{0}\right)$.

\section{Numerical simulations}

The numerical simulations of a 2D strip under uniaxial compression are carried out in this section. The material properties and model parameters are as follows: $E=60 \mathrm{GPa}, v$ $=0.2, \sigma_{\mathrm{c} 0}=100 \mathrm{MPa}, \rho=2600 \mathrm{~kg} / \mathrm{m}^{3}, \psi=\varphi=45^{\circ}, K_{\mathrm{m}}=-\sigma_{\mathrm{c} 0}{ }^{2} h_{\mathrm{e}} /\left(2 G_{\text {IIc }}\right), G_{\text {IIc }}=2.5$ $\mathrm{J} / \mathrm{mm}^{2}, \alpha=1 \mathrm{E}-5, \mathrm{~K}^{-1}, c=700 \mathrm{~J} / \mathrm{kgK}, K_{\theta}=-0.467 \mathrm{MPa} / \mathrm{K}, K_{\theta}{ }^{E}=-143 \mathrm{MPa} / \mathrm{K}$. Here, $h_{\mathrm{e}}$ $=\left(2 A_{e}\right)^{1 / 2}$ is the characteristic size of an element in the mesh, and $\alpha$ is the thermal expansion coefficient. The boundary conditions are illustrated in Figure 1a. The shear band is triggered by a weak element at the left upper corner of the mesh, which consists of 200 bi-linear rectangular (square here) elements. The first simulation is carried out at $\dot{\varepsilon}=1 \mathrm{~s}^{-1}$ with $s=0.01 \mathrm{MPas}$. The results are shown in Figure 1 .

According to the simulation results in Figure 1b, the equivalent viscoplastic strain in the shear band exceeds 0.2. This quite intensive deformation is illustrated in Figure 1d with a magnification factor of 5 . However, the consequent temperature rise in the shear band at the end softening process is less than 1.5 degrees (see Figure 1c and f). Finally, the compressive strength of the specimen in this test is $100 \mathrm{MPa}$ as expected. Next the same problem is simulated at $\dot{\varepsilon}=10 \mathrm{~s}^{-1}$ with $s=0.5 \mathrm{MPas}$. The results are shown in Figure 2.

At higher strain rate with higher viscosity some strain rate hardening occurred, as can be observed in Figure 2a, where the maximum average stress is $120 \mathrm{MPa}$. Moreover, practically the whole specimen has undergone some viscoplastic deformation, as attested in Figure $2 \mathrm{~b}$. Temperature rise is still somewhat mild exceeding barely 1.5 degrees (centigrade). With this temperature change then, the decrease in tensile strength and the Young's modulus are $-0.72 \mathrm{MPa}$ and $-0.21 \mathrm{GPa}$, respectively. These values, being $0.72 \%$ and $0.36 \%$ in percent, are quite insignificant so that assuming this problem to be isothermal is justified. 


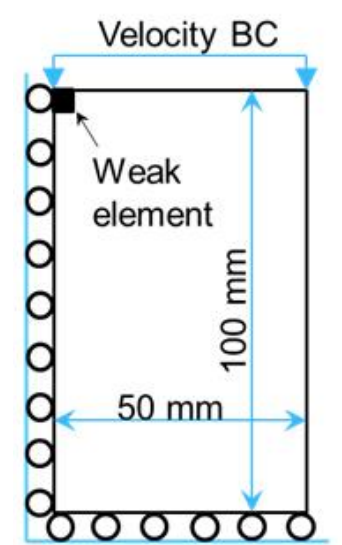

a

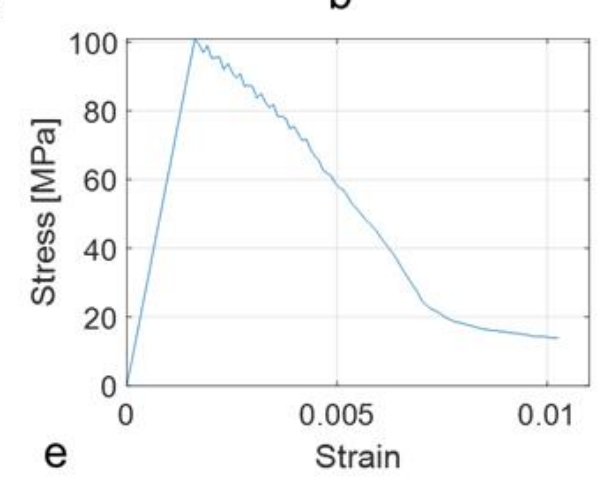

b
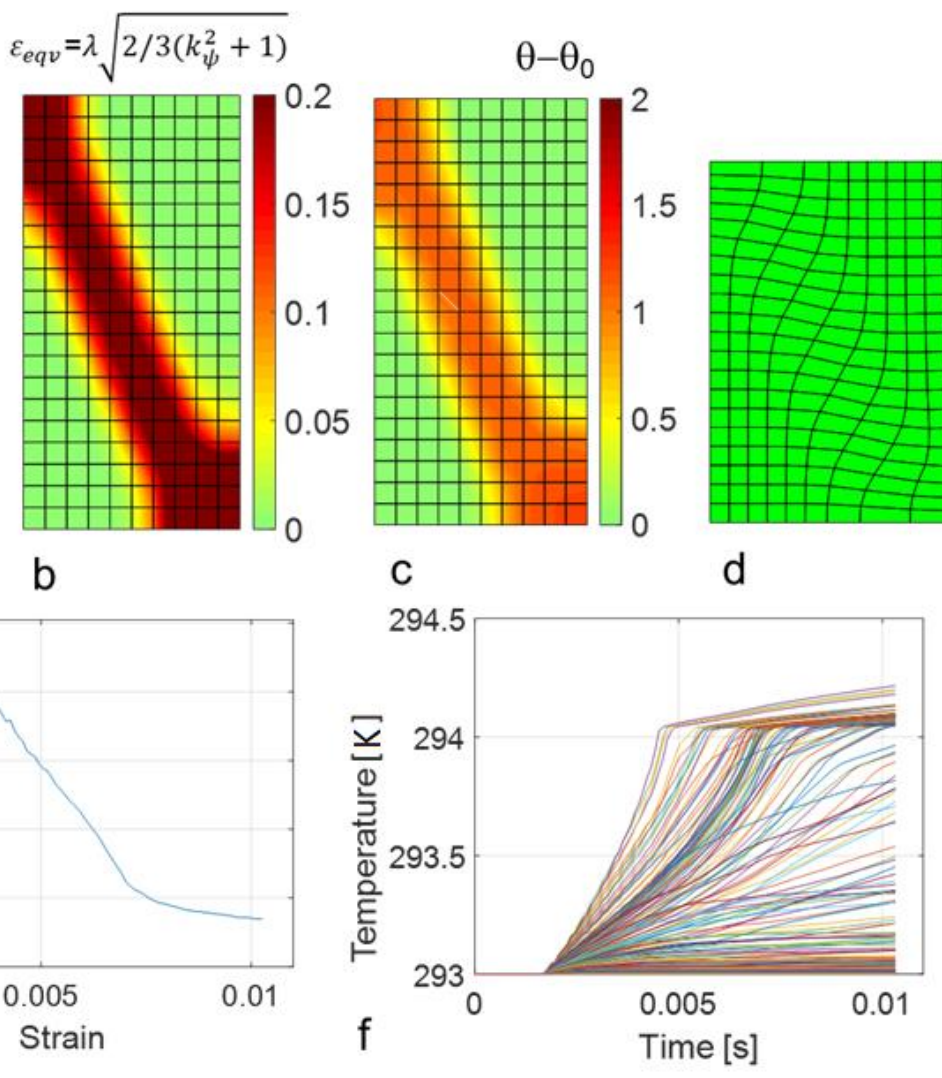

Figure 1. Simulation results at strain rate $1 / \mathrm{s}$ with $s=0.01$ MPas: Geometry and boundary conditions (a), final shear band in terms of equivalent viscoplastic strain (b), temperature change at the end of simulation (c), deformed geometry (magnification $=5)(d)$, stress-strain curve (e), and the nodal temperatures as a function of time (f).

\section{Conclusions}

A numerical scheme to solve the problem of adiabatic heating due to viscoplastic shear band formation in rock like materials was developed and applied in simulations of a 2D strip under dynamic uniaxial compression. The problem reduced into solving a second order polynomial for the viscoplastic increment during stress integration at an integration point. This result is generally valid only for the specific case studied in this paper, i.e. Mohr-Coulomb viscoplasticity with only the compressive strength assumed to depend on temperature. In more general cases, the Newton-Raphson iteration must be used.

According to the simulations of controlled shear band formation in a rock under dynamic uniaxial compression, the temperature rise is less than a couple of degrees up to $10 / \mathrm{s}$ of strain rate. Therefore, the usual isothermal assumption is justified. 


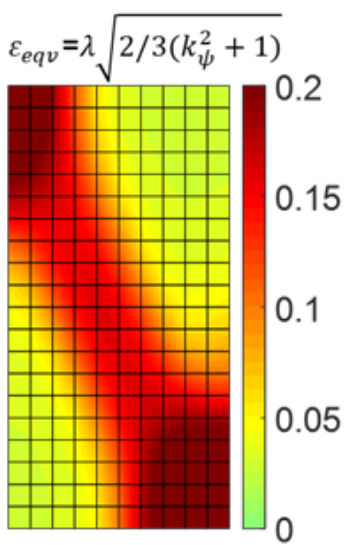

a

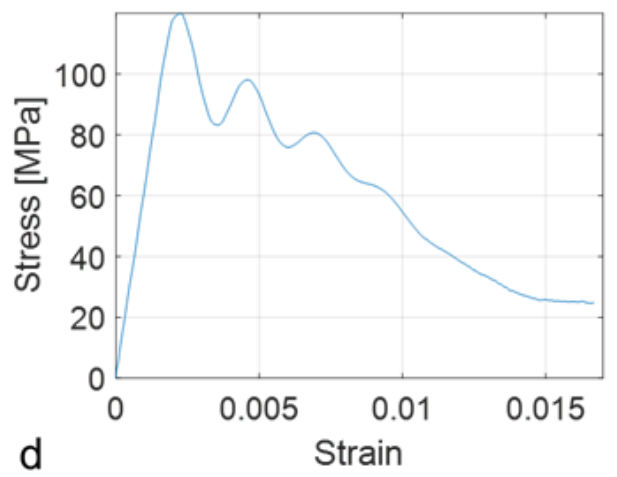

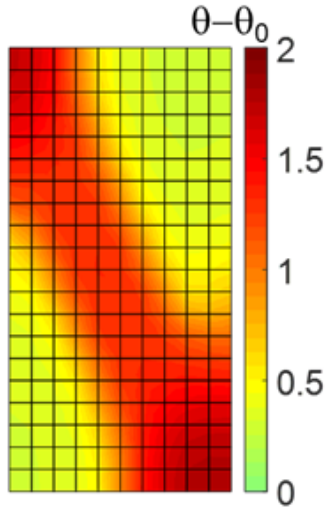

b

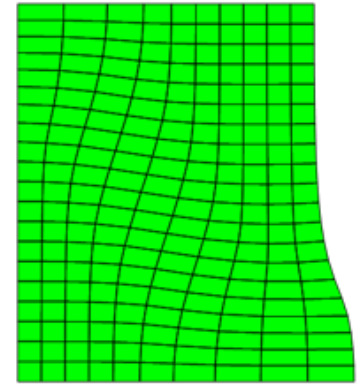

C

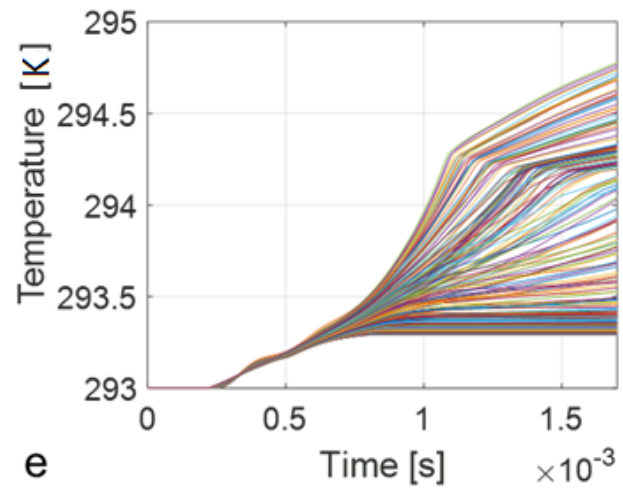

Figure 2. Simulation results at strain rate 10/s with $s=0.5$ MPas: Final shear band in terms of equivalent viscoplastic strain (a), temperature change at the end of simulaton (b), deformed geometry (magnification $=5$ ) $(\mathrm{c})$, stress-strain curve $(\mathrm{d})$, and the nodal temperatures as a function of time (e).

\section{Acknowledgements}

This research was funded by Academy of Finland under Grant number 298345.

\section{References}

[1] Y. Ben-Zion, C.G. Sammis. Shear heating during distributed fracturing and pulverization of rocks. GEOLOGY, 41(2): 139-142, 2013. https://doi.org/10.1130/G33665.1

[2] N.S. Ottosen, M. Ristinmaa. The Mechanics of Constitutive Modeling. Elsevier: Amsterdam, 2005.

[3] W.M. Wang, L.J. Sluys, R. De Borst. Viscoplasticity for instabilities due to strain softening and strain-rate softening. International Journal for Numerical Methods in Engineering, 40:3839-3864, 1997. https://doi.org/10.1002/(SICI)10970207(19971030)40:20<3839::AID-NME245>3.0.CO;2-6

[4] J.C. Simo, T.J.R. Hughes. Computational Inelasticity. Springer: Berlin, 1998. 
Timo Saksala, Martina Pressacco, Sami Holopainen, Reijo Kouhia Tampere University, Civil Engineering, P.O. Box 600, FI-33101 Tampere, Finland timo.saksala@tuni.fi,martina.pressacco@tuni.fi,sami.holopainen@tuni.fi, reijo.kouhia@tuni.fi 